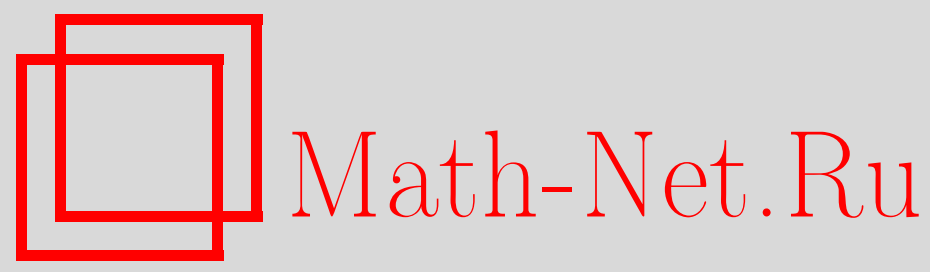

В. В. Козлов, Д. В. Трещёв, О неустойчивости изолированных равновесий динамических систем с инвариантной мерой в нечетномерном пространстве, Матем. заметки, 1999, том 65, выпуск 5, 674-680

DOI: https://doi.org/10.4213/mzm1099

Использование Общероссийского математического портала Math-Net.Ru подразумевает, что вы прочитали и согласны с пользовательским соглашением http://www.mathnet.ru/rus/agreement

Параметры загрузки:

IP : 54.209 .52 .79

26 апреля 2023 г., $15: 27: 22$

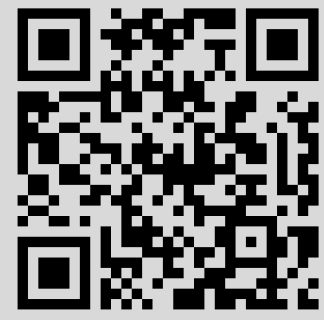




\title{
О НЕУСТОЙЧИВОСТИ ИЗОЛИРОВАННЫХ РАВНОВЕСИЙ ДИНАМИЧЕСКИХ СИСТЕМ С ИНВАРИАНТНОЙ МЕРОЙ В НЕЧЕТНОМЕРНОМ ПРОСТРАНСТВЕ
}

\author{
В.В. Козлов, Д. В. Трещев
}

\begin{abstract}
В работе обсуждается гипотеза о неустойчивости изолированных положений равновесия автономных систем в пространстве нечетной размерности, допускающих инвариантную меру. Эта гипотеза доказана для систем, у которых можно выделить квазиоднородное укорочение с изолированной особенностью. Приведен контрпример в классе систем с бесконечно дифференцируемой правой частью и нулевым рядом Маклорена в положении равновесия.

Библиограффия: 3 названия.
\end{abstract}

1. Введение. Пусть

$$
\dot{x}=v(x), \quad x \in \mathbb{R}^{n},
$$

- автономная система дифференциальных уравнений, допускающая инвариантную меру с гладкой плотностью:

$$
\operatorname{div}(\rho v)=0, \quad x \in \mathbb{R}^{n} .
$$

Пусть $x=0$ - положение равновесия: $v(0)=0$.

В. В. Тен высказал предположение, что если $n$ - нечетно и равновесие $x=0$ изолировано, то оно неустойчиво по Ляпунову. Эта гипотеза имеет важное следствие: все изолированные положения равновесия стационарных течений жидкости в трехмерном евклидовом пространстве неустойчивы.

В типичной ситуации система (1) имеет следуюший вид:

$$
\dot{x}=A x+o(|x|), \quad \operatorname{det} A \neq 0 .
$$

Перепишем “уравнение неразрьвности” (2): $\dot{\nu}=-\operatorname{div} v$, где $\nu=\ln \rho$. Полагая в этом уравнении $x=0$, получаем равенство $\operatorname{tr} A=0$. Следовательно, сумма всех собственных значений матрищы $A$ равна нулю. Хотя бы одно из собственных значений лежит в правой полуплоскости. Действительно, в противном случае спектр матрицы $A$ расположен на мнимой оси. Поскольку $A$ - вешественная матрища, сумма всех ее собственных значений равна нулю и $n$ нечетно, то нуль обязательно будет собственным значением. Однако

Работа выполнена при поддержке Российского фонда фундаментальных исследований, грант № 96-01-00747 и INTAS. 
это противоречит предположению о невырожденности матрицы $A$. Следовательно, по теореме Ляпунова $x=0$ - неустойчивое равновесие системы (3).

Аналогично доказьвается, что невырожденная периодическая траектория динамической системы с инвариантной мерой в четномерном пространстве всегда неустойчива. Напомним, что периодическая орбита назьвается невырожденной, если ее мультипликаторы отличны от единищы. Это наблюдение справедливо и для невырожденных приводимых инвариантных торов нечетной коразмерности, заполненных условно-периодическими траекториями.

2. Полуквазиоднородные системы. Это утверждение о неустойчивости можно распространить на системы с полуоднородной правой частью:

$$
v=v_{m}+v_{m+1}+\cdots, \quad \text { где } \quad v_{k}(\lambda x)=\lambda^{k} v_{k}(x), \quad m \geq 1
$$

Единственное дополнительное условие состоит в том, что $x=0$ - единственное равновесие однородного поля $v_{m}$.

Мы рассмотрим даже более общий случай полуквазиоднородного векторного поля. Напомним, что поле $v(x)$ назьвается квазиоднородным полем степени $m$ с показателями квазиоднородности $g_{1}, \ldots, g_{n}>0$, если

$$
v_{i}\left(\lambda^{g_{1}} x_{1}, \ldots, \lambda^{g_{n}} x_{n}\right)=\lambda^{g_{i}+m-1} v_{i}\left(x_{1}, \ldots, x_{n}\right),
$$

где $v_{i}-i$-я компонента поля $v$. Гладкое поле $v$ называется полуквазиоднородным, если его можно представить в виде формального ряда

$$
v_{m}+\sum_{\alpha>m} v_{\alpha}
$$

где $v_{k}-$ квазиоднородные поля степени $k$ с одними и теми же показателями квазиоднородности. Для однородных полей можно положить $g_{1}=\cdots=g_{m}=1$.

Поле $v_{m}$ назьвается квазиоднородным укорочением исходного поля $v$. Отметим, что для одной и той же системы можно по-разному выбирать квазиоднородные укорочения. Поясним это на примере системы на плоскости

$$
\dot{x}_{1}=x_{2}^{2}, \quad \dot{x}_{2}=x_{1}^{3}
$$

Если принять $g_{1}=g_{2}=1$, то квазиоднородньм укорочением будет система $\dot{x}_{1}=x_{2}^{2}$, $\dot{x}_{2}=0$. Если $g_{1}=3 / 5, g_{2}=4 / 5$, то квазиоднородное укорочение совпадает с исходной системой (5). В первом случае начало координат $x_{1}=x_{2}=0$ - неизолированное положение равновесия, а во втором случае оно изолировано. Алгоритмы выделения квазиоднородных укорочений обсуждаются в [1].

ТЕорема 1. Предположим, что поле $v$ можсно представить в виде (4), причем $x=0$ - изолированный нуль поля $v_{m}$. Если $n$ нечетно и система допускает инвариантную меру, то равновесие $x=0$ неустойчиво. 
ДоКАЗАТЕЛЬСТВО. Поскольку $n$ нечетно и $x=0$ - единственный нуль квазиоднородного поля $v_{m}$, то (как показанов [2]) найдется ненулевой вектор $z$, удовлетворяющий одному из уравнений

$$
v_{m}(z)=-G z \quad \text { или } \quad v_{m}(z)=G z,
$$

где $G=\operatorname{diag}\left(g_{1}, \ldots, g_{n}\right)$ - диагональная матрища. Как установлено в [2], в этом случае уравнения (1) допускают решения с асимптотикой

$$
z t^{-G} \quad \text { или } \quad z t^{-G}
$$

соответственно при $t \rightarrow+\infty$ или $t \rightarrow-\infty$. Эти решения стремятся к равновесию $x=0$ при $t \rightarrow \pm \infty$. Если имеется решение второго вида (“выходяшее из точки $x=0$ ”), то, очевидно, равновесие $x=0$ неустойчиво. Осталось рассмотреть случай, когда имеется решение, асимптотическое к равновесию $x=0$ при $t \rightarrow+\infty$.

Воспользуемся утверждением, представляюшим самостоятельный интерес.

ЛЕмма 1. Предположим, что система (1) с инвариантной мерой допускает нетривиальное решение $t \mapsto x(t)$, которое стремится $\kappa$ нулю при $t \rightarrow+\infty$. Тогда равновесие $x=0$ неустойчиво.

Действительно, пусть точка $x_{0}=x(0)$ лежит вне некоторой $\varepsilon_{0}$-окрестности нуля. Для любого $\varepsilon>0$ найдется малая окрестность $U$ точки $x_{0}$, которая под действием фазового потока через некоторое время целиком окажется в $\varepsilon$-окрестности точки $x=0$. Так как фазовьй поток сохраняет меру, по теореме Шварцшильда-Литтлвуда [3] почти все траектории с начальными условиями из $U_{\varepsilon}$ покинут $\varepsilon_{0}$-окрестность точки $x=0$. Это доказьвает неустойчивость равновесия $x=0$, поскольку выходяшие траектории пересекаются с $\varepsilon$-окрестностью нуля.

3. Контрпример для гладкого случая. Покажем теперь, что гипотеза Тена не справедлива в гладком случае без каких-либо дополнительных предположений о невырожденности.

Во-первых, отметим, что достаточно иметь пример в $\mathbb{R}^{3}$, так как, имея пример в $\mathbb{R}^{2 n+1}$, с помошью прямого произведения на систему

$$
\dot{x}=-y, \quad \dot{y}=x, \quad(x, y) \in \mathbb{R}^{2},
$$

получаем пример в $\mathbb{R}^{2 n+3}$.

Согласно построению окажется, что в достаточно малой окрестности равновесия нет периодических решений с периодами, меньшими любой наперед заданной постоянной. Отсюда следует, что, добавляя уравнение $\dot{\psi}=1, \psi \in \mathbb{R} /(2 \pi \mathbb{Z})$, получаем пример устойчивого изолированного периодического решения для бездивергентного векторного поля в четномерном пространстве.

Сначала опишем основную идею. Мы построим последовательность $\mathbb{R}^{3} \supset S_{0} \supset$ $S_{1} \supset \cdots$ вложенных замкнутых полноторий, диаметр которых как множеств в $\mathbb{R}^{3}$ стремится к нулю. Векторное поле $v$ определено на $S_{0}$ и имеет единственную особую точку $O=\bigcap_{n=0}^{\infty} S_{n}$. Так как границы полноторий $\sigma_{k}=\partial S_{k}$ будут инвариантньми относительно $v$, особая точка $O$ устойчива по Ляпунову.

Излагаемая конструкция индуктивна. Поэтому достаточно определить векторное поле $v_{0}$ - ограничение $v$ на область $S_{0} \backslash S_{1}$. С помошью диффеоморфизма $F$ отобразим 
эту область в пространство $\mathbb{R}^{3} / G$, где $G$ - группа параллельных переносов $(r, \varphi, h) \mapsto$ $(r, \varphi, h+2 \pi k), k \in \mathbb{Z}$. Здесь $(r, \varphi, h)$ - цилиндрические координаты в $\mathbb{R}^{3}$. Пространство $\mathbb{R}^{3} / G$ диффеоморфно прямому произведению двумерной плоскости на окружность. Ниже удобно представлять себе $\mathbb{R}^{3} / G$ как полосу $\{(r, \varphi, h):-\pi \leq h \leq \pi\}$, в которой нижняя и верхняя плоскости отождествлены. Тор $M=F\left(\sigma_{0}\right)$ имеет вид $M=\{(r, \varphi, h)$ : $r=a\}$. Будем считать, что

$$
F\left(\sigma_{1}\right)=N=\left\{(r, \varphi, h):(r-a / 2)^{2}+h^{2}=a^{2} / 16\right\}
$$

Область $D=F\left(S_{0} \backslash S_{1}\right)$ заключена между торами $M$ и $N$ (см. рис. 1$)$.

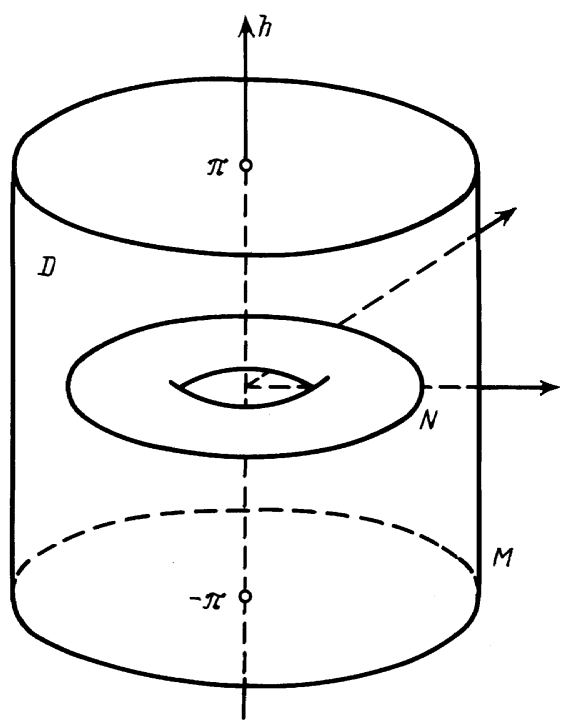

Рис. 1. Область $D$

Поверхности $M$ и $N$ инвариантны относительно вращений вокруг оси $h$. Векторное поле $u=F_{*}\left(v_{0}\right)$ также будет инвариантньм относительно врашений. Мы положим $u=$ $u^{\prime}+u^{\prime \prime}$, где $u^{\prime}=\partial / \partial \varphi$, а $u^{\prime \prime}$ касается торов $M, N$ “вертикальных цилиндров" $\{(r, \varphi, h)$ : $\varphi=$ const $\}$. Потребуем, чтобы $u^{\prime \prime}$ имело положительную проекцию на ось $h$ в точках окружности $\{(r, \varphi, h): r=0\}$. Тогда векторное поле $u$ не имеет особых точек на $D$. Поле $u^{\prime \prime}$ можно сделать бездивиргентньм.

Векторные поля $v_{k}=\left.v\right|_{S_{k} \backslash S_{k+1}}, k>0$, строятся аналогично. Остается гладко склеить поля $v_{k}$ и $v_{k+1}$, а также позаботиться о том, чтобы $\left|v_{k}\right|$ достаточно быстро стремились к нулю при $k \rightarrow \infty$.

Теперь перейдем к аккуратному построению. Чтобы добиться гладкости, конструкцию придется несколько усложнить. А именно, последовательность полноторий будет иметь вид

$$
\mathbb{R}^{3} \supset S_{0} \supset T_{1} \supset S_{1} \supset T_{2} \supset \cdots
$$


Все торы $\sigma_{k}=\partial S_{k}, \tau_{k}=\partial T_{k}$ будут инвариантньми. Описанная вьше последовательность векторных полей $v_{k}$ определена на последовательности областей $S_{k} \backslash T_{k+1}$, а в областях $T_{k+1} \backslash S_{k+1}$, диффеоморфных прямому произведению двумерного тора на полуинтервал, производится гладкий переход от $v_{k}$ к $v_{k+1}$.

Опишем подробнее основной элемент конструкции - векторное поле $v_{k}$. Сначала построим его прообраз - векторное поле $u=u^{\prime}+u^{\prime \prime}$ в области $D$. Поле $u^{\prime}$ уже построено. Оно сохраняет стандартный объем на $\mathbb{R}^{3} / G$. Поле $u^{\prime \prime}$ достачно построить на цилиндре

$$
Z=\{(r, \varphi, h): \varphi=0,0<r<2 a\}
$$

В определении цилиндра $Z$ мы пишем $r<2 a$ вместо $r<a$, так как далее удобно предполагать, что $u$ гладко продолжается в окрестность замыкания $\bar{D}$ области $D$. Стандартная форма объема на $\mathbb{R}^{3}$ имеет вид $r d r \wedge d \varphi \wedge d h$. Поэтому условие сохранения объема полем $u^{\prime \prime}$ переходит в условие сохранения формы площади $r d r \wedge d h$ на $Z$.

Рассмотрим гладкую функцию $H: Z \rightarrow \mathbb{R}$ такую, что

i) $H=r^{2}$ в окрестности окружности $\{r=0\}$,

ii) $H=r^{2}+$ const в окрестности окружности $\{(r, \varphi, h): \varphi=0, r=a\}$,

iii) функция $H$ постоянна на окружности

$$
\Lambda=\left\{(r, \varphi, h): \varphi=0,(r-a / 2)^{2}+h^{2}=a^{2} / 16\right\} .
$$

Возьмем в качестве $\left.u^{\prime \prime}\right|_{Z}$ гамильтоново векторное поле с гамильтонианом $H$ в симплектической структуре $r d r \wedge d h$. Очевидно, $\left.u^{\prime \prime}\right|_{Z}$ сохраняет форму $r d r \wedge d h$, при малых $r$ и при $r$ близких к $a$ имеем $\left.u^{\prime \prime}\right|_{Z}=2 \partial / \partial h$; кроме того, $\left.u^{\prime \prime}\right|_{Z}$ касается окружностей $\{r=a\}$ и $\Lambda$. Итак, векторное поле $u$ на области $D$ (и даже на окрестности ее замыкания) построено. Оно сохраняет объем и не имеет особых точек. Действительно, вне окружности $\{r=0\}$ проекция $u$ на плоскость $\{h=0\}$ отлична от нуля, а на окружности $\{r=0\}$ имеем: $u=u^{\prime \prime}=2 \partial / \partial h$.

Далее величину $a$ в определении области $D$ будем считать малой.

Рассмотрим отображение $f: D \rightarrow \mathbb{R}^{3}$, задаваемое формулами $f(r, \varphi, h)=(x, y, z)$, где

$$
\begin{aligned}
& x=(1+\rho \cos \varphi) \cos h, \\
& y=(1+\rho \cos \varphi) \sin h, \\
& z=\rho \sin \varphi .
\end{aligned}
$$

Здесь $\rho=\rho(r, \varphi)$ - наименьший неотрицательньй корень уравнения

$$
\rho^{2}+\frac{2}{3} \rho^{3} \cos \varphi=r^{2}
$$

Так как $r$ принимает значения на отрезке [0,a] (или при необходимости продолжения отображения $f$ на большую область - на отрезке $[0, a])$, где $a>0$ мало, функция $\rho(r, \varphi)$ гладкая.

В результате прямых вычислений получаем:

$$
d x \wedge d y \wedge d z=\left(\rho+\rho^{2} \cos \varphi\right) d \rho \wedge d \varphi \wedge d h=r d r \wedge d \varphi \wedge d h
$$


т.е. отображение $f$ сохраняет объем. Следовательно, векторное поле $v_{0}=f_{*} u$ сохраняет стандартньй объем на области $f(D)$.

Положим $\sigma_{0}=f(M), \tau_{1}=f(N)$. Из условия малости $a$ следует, что тор $\sigma_{0}$ близок к окружности

$$
\lambda_{0}=\{(x, y, z): x=\cos h, y=\sin h, z=0\} .
$$

С другой стороны, если величина $а$ мала, то окружность

$$
\lambda_{1}=\left\{(x, y, z): x=1+\frac{a}{2} \cos \varphi, y=0, z=\frac{a}{2} \sin \varphi\right\}
$$

лежит внутри тора $\tau_{1}$. Следовательно, внутри $\tau_{1}$ лежит некоторьй тор, подобньй тору $\sigma_{0}$ и близкий к окружности $\lambda_{1}$.

Замкнутые полнотория с границами $\sigma_{0}, \tau_{1}, \sigma_{1}$ обозначим $S_{0}, T_{1}, S_{1}$ соответственно. Очевидно, $S_{1} \subset T_{1} \subset S_{0}$. Область $T_{1} \backslash S_{1}$ диффеоморфна двумерному тору, умноженному на полуинтервал.

Пусть $g: \mathbb{R}^{3} \rightarrow \mathbb{R}^{3}$ - преобразование подобия, переводящее $\sigma_{0}$ в $\sigma_{1}$. Положим

$$
\begin{aligned}
& \tau_{2}=g\left(\tau_{1}\right), \quad \sigma_{2}=g\left(\sigma_{1}\right), \quad \tau_{3}=g\left(\tau_{2}\right), \quad \ldots, \\
& T_{2}=g\left(T_{1}\right), \quad S_{2}=g\left(S_{1}\right), \quad T_{3}=g\left(T_{2}\right), \ldots
\end{aligned}
$$

Векторные поля $v_{k}=\left.v\right|_{S_{k} \backslash T_{k+1}}$ возьмем следуюшими:

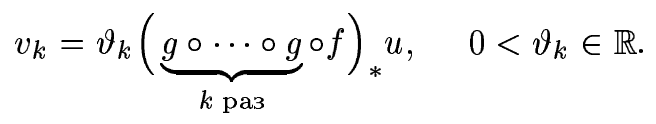

Итак, поле $v$ определено в объединении областей $\left(S_{0} \backslash T_{1}\right) \cup\left(S_{1} \backslash T_{2}\right) \cup \cdots$.

Достроить $v$ до гладкого поля в области $S_{0}$ не представляет труда. Действительно, окрестность области $T_{k} \backslash S_{k}$ некоторым диффеоморфизмом $Q_{k}$ переводится в область

$$
\{(\mu, \alpha, \beta):-\varepsilon<\mu<1+\varepsilon, \alpha \bmod 2 \pi, \beta \bmod 2 \pi\}, \quad 0<\varepsilon<\frac{1}{3},
$$

причем

$$
Q_{k}\left(\tau_{k}\right)=\{(\mu, \alpha, \beta): \mu=0\}, \quad Q_{k}\left(\sigma_{k}\right)=\{(\mu, \alpha, \beta): \mu=1\} .
$$

Более того, можно считать, что ограничение поля $\left(Q_{k}\right)_{*} v$ на торы $Q_{k}\left(\tau_{k}\right)$ и $Q_{k}\left(\sigma_{k}\right)$ имеет положительную координату при $\partial / \partial \alpha$ в базисе $\partial / \partial \mu, \partial / \partial \alpha, \partial / \partial \beta$. Существование гладкого векторного поля, совпадающего с $\left(Q_{k}\right)_{*} v$ в областях $\{(\mu, \alpha, \beta):-\varepsilon<\mu<0\}$ и $\{(\mu, \alpha, \beta): 1<\mu<1+\varepsilon\}$, вытекает из возможности гладкого продолжения $\left(Q_{k}\right)_{*} v$ на области $\{(\mu, \alpha, \beta): \varepsilon<\mu<\varepsilon\}$ и $\{(\mu, \alpha, \beta): 1-\varepsilon<\mu<1+\varepsilon\}$.

Гладкость в положении равновесия $O$ достигается достаточно быстрым стремлением последовательности $\vartheta_{k}$ к нулю. При этом производные всех порядков поля $v$ в точке $O$ будут нулевьми. Одновременно получаем отсутствие в окрестности точки $O$ периодических решений малых (например, не превосходящих $2 \pi$ ) периодов.

Не исключено, что обсуждаемая гипотеза справедлива в аналитическом случае.

4. Потенциальные поля. Имея в виду приложения к гидродинамике, рассмотрим потенциальное векторное поле (1): $v=\partial \varphi / \partial x$. 
Теорема 2. Пусть ч - непостоянная аналитическая функиия. Тогда все равновесия системы (1) неустойчивы.

Подчеркнем, что в этом утверждении нет предположения о нечетности размерности $n$.

ДокАЗАТЕЛЬСТво. Пусть $x=0$ - критическая точка функции $\varphi$ и $\varphi(0)=0$. Разложим потенциал в ряд Маклорена по однородным формам:

$$
\varphi=\varphi_{m}+\varphi_{m+1}+\cdots
$$

Поскольку $\varphi \not \equiv$ const, то $\varphi_{m} \not \equiv 0$ при некотором $m \geq 2$. Разложим плотность инвариантной меры в ряд Маклорена: $\rho=\rho_{0}+\cdots, \rho_{0}=$ const $>0$. Из уравнения $(2)$ вытекает, что $\varphi_{m}$ - гармоническая функция. Ввиду теоремы о среднем она принимает значения разных знаков на единичной сфере $S^{n-1}=\{x:\|x\|=1\}$. Пусть $z$ - точка максимума функции $\varphi_{m}$ на $S^{n-1}$; очевидно, $\varphi_{m}(z)>0$. Согласно правилу множителей Лагранжа в точке $x=z$

$$
\frac{\partial \varphi_{m}}{\partial x}=\mu x .
$$

По формуле Эйлера для однородных функций

$$
m \varphi_{m}=\left(\frac{\partial \varphi_{m}}{\partial x}, x\right)=\mu(x, x)=\mu .
$$

Следовательно, $\mu>0$. Итак, алгебраическое уравнение $v_{m-1}(x)=\mu x$ $\left(v_{m-1}(x)=\partial \varphi_{m} / \partial x\right)$ имеет нетривиальное решение с $\mu>0$. Следовательно, согласно [2] исходная система (1) допускает асимптотическое решение, "выходяшее" из положения равновесия $x=0$. Теорема доказана.

Авторы дружески благодарят В. В. Тена за полезные обсуждения.

\section{СПИСОК ЦИТИРОВАННОЙ ЛИТЕРАТУРЫ}

[1] Брюно А. Д. Степенные асимптотики решений нелинейных систем // Изв. АН СССР. Сер. матем. 1965. Т. 29. № 2. С. 329-364.

[2] Козлов В.В., Фурта С.Д. Первый метод Ляпунова для сильно нелинейных систем // ПММ. 1996. Т. 60. № 1. С. 10-22.

[3] Зигель К. Л. Лекции по небесной механике. М.: ИЛ, 1959.

Московский государственный университет им. М.В.Ломоносова Поступило 21.05 .98 